\title{
ON THE BACKWARD UNIQUENESS PROPERTY FOR A CLASS OF PARABOLIC OPERATORS
}

\author{
DANIELE DEL SANTO AND MARTINO PRIZZI
}

\begin{abstract}
We give sharp regularity conditions, ensuring the backward uniquess property to a class of parabolic operators.
\end{abstract}

\section{InTRODUCTION, STATEMENTS AND REMARKS}

In this note we illustrate some new results concerning the backward uniqueness property for a class of parabolic operators, whose coefficients are non-Lipschitz continuous in time. Namely, we consider parabolic operators of the form

$$
P:=\partial_{t}+\sum_{0 \leq|\alpha|,|\beta| \leq m}(-1)^{|\alpha|} \partial_{x}^{\alpha}\left(\rho_{\alpha \beta}(t, x) \partial_{x}^{\beta}\right) ;
$$

here $m \in \mathbb{N},(t, x) \in[0, T] \times \mathbb{R}^{n}$, and $\alpha$ and $\beta$ are $n$-multiindices with weights $|\alpha|$ and $|\beta| \leq m$. We assume that $\rho_{\alpha \beta}=\overline{\rho_{\beta \alpha}}$ for all $\alpha$ 's and $\beta$ 's (formal self-adjointness), that $\rho_{\alpha \beta}$ is real when $|\alpha|=|\beta|=m$, and that there exists $c_{0}>0$ such that $\sum_{|\alpha|=|\beta|=m} \rho_{\alpha \beta}(t, x) \xi^{\alpha} \xi^{\beta} \geq c_{0}|\xi|^{2 m}$ for all $\xi \in \mathbb{R}^{n}$ (strong ellipticity).

Given a functional space $\mathcal{H}$, we say that $P$ enjoys the backward uniqueness property in $\mathcal{H}$ iff, whenever $u \in \mathcal{H}$ satisfies $P u \equiv 0$ (in the sense of distributions) in $[0, T] \times \mathbb{R}^{n}$, and $u(T, \cdot) \equiv 0$ in $\mathbb{R}^{n}$, then $u \equiv 0$ in $[0, T] \times \mathbb{R}^{n}$. Our aim is to find conditions on the coefficients $\rho_{\alpha \beta}$ 's, ensuring that $P$ enjoys the backward uniqueness property in some given functional space $\mathcal{H}$. As a preliminary observation, we notice that in [13] Tychonoff constructed a function $u \in C^{\infty}\left(\mathbb{R} \times \mathbb{R}^{n}\right)$ satisfying

$$
\begin{cases}\partial_{t} u-\Delta u \equiv 0 & \text { in } \mathbb{R} \times \mathbb{R}^{n} \\ u(0, \cdot) \equiv 0 & \text { in } \mathbb{R}^{n}\end{cases}
$$

but $u \not \equiv 0$ in any open subset of $\mathbb{R} \times \mathbb{R}^{n}$. It follows that, whether $P$ enjoys the backward uniqueness property in $\mathcal{H}$ or not, depends first of all on the choice of $\mathcal{H}$. We are interested here in the case $\mathcal{H}=\mathcal{H}_{1}^{m}$, where

$$
\mathcal{H}_{1}^{m}:=H^{1}\left([0, T], L^{2}\left(\mathbb{R}^{n}\right)\right) \cap L^{2}\left([0, T], H^{2 m}\left(\mathbb{R}^{n}\right)\right) .
$$

The reason for this choice is essentially due to its historical background, but other choices are possible as well. In [7] Lions and Malgrange proved that $P$ enjoys the backward uniqueness property in $\mathcal{H}_{1}^{m}$, provided the $\rho_{\alpha \beta}$ 's are sufficiently smooth with

Date: November 19, 2018.

1991 Mathematics Subject Classification. 35K10, 35B40 .

Key words and phrases. parabolic operator, backward uniqueness, modulus of continuity, Osgood condition. 
respect to $x$ and Lipschitz continuous with respect to $t$. They work in an abstract Hilbert space setting and their proof is based on a Carleman type estimate. The required smoothness of the $\rho_{\alpha \beta}$ 's with respect to $x$ is related to the regularity theory for elliptic equations and is needed to let $P$ fall in the abstract Hilbert space setting. The required Lipschitz continuity with respect to $t$ seems to be more intrinsically connected with the backward uniqueness property. Infact, in the same paper Lions and Malgrange raised the question, whether Lipschitz continuity could be replaced by, say, simple continuity. As a first step in this direction, in [3] Bardos and Tartar proved that $P$ enjoys the backward uniqueness property in $\mathcal{H}_{1}^{m}$, provided the $\rho_{\alpha \beta}$ 's are absolutely continuous with respect to $t$. Their proof exploits a sort of logarithmic convexity property satisfied by the norm $\|u\|$ of any nontrivial solution of $P u=0$. Later, in [6] Ghidaglia, by using the same technique, extended the results of [3], so as to cover also some classes of nonlinear parabolic equations. We stress that, in all the above mentioned results, it is required that the $\rho_{\alpha \beta}$ 's be differentiable with respect to $t$, at least in a weak sense. The reason is that, at a certain point, one needs to perform some integration by parts. Although this latter seems to be just a technical obstruction, the possibility of replacing Lipschitz continuity by simple continuity was finally ruled out by Miller in [9]. He exibited an example of an operator $P$ which does not enjoy the backward uniqueness property in $\mathcal{H}_{1}^{m}$. The operator is of second order in space and its coefficients are of class $C^{\infty}$ with respect to $x$ and Hölder continuos of exponent $1 / 6$ with respect to $t$. Recently, in [8], Mandache improved the result of Miller constructing a similar non-uniqueness example in which the coefficients are of class $C^{\infty}$ with respect to $x$ and Hölder continuos of every exponent less than 1 with respect to $t$. More precisely in the result of Mandache the regularity with respect to $t$ is expressed in terms of a modulus of continuity. Our goal is to find a sharp condition on the modulus of continuity of the $\rho_{\alpha \beta}$ 's, ensuring that $P$ enjoys the backward uniqueness property in $\mathcal{H}_{1}^{m}$.

Let $I \subset \mathbb{R}$ be a closed bounded interval, let $\mathcal{B}$ be a Banach space and let $f: I \rightarrow \mathcal{B}$ be a continuous function. The modulus of continuity of $f$ is the function $\mu(f, \cdot):[0,1] \rightarrow \mathbb{R}$ defined by

$$
\mu(f, \tau):=\sup _{\substack{t, s \in I \\ 0 \leq|t-s| \leq \tau}}\|f(t)-f(s)\|_{\mathcal{B}}
$$

Notice that $\mu(f, \cdot)$ is nondecreasing and $\mu(f, 0)=0$. Since $f$ is uniformly continuous on $I$, it follows that $\mu(f, \tau) \rightarrow 0$ as $\tau \rightarrow 0$. If $f$ is nonconstant, then $\mu(f, \tau)>0$ for $\tau>0$ and $\mu(\cdot)=\mu(f, \cdot)$ satisfies

$$
\sup _{\substack{t, s \in I \\ 0<|t-s| \leq 1}} \frac{\|f(t)-f(s)\|_{\mathcal{B}}}{\mu(|t-s|)}<+\infty .
$$

Moreover, $\mu(f, \cdot)$ is minimal with respect to this latter property, in the sense that, whenever a nondecreasing function $\mu:[0,1] \rightarrow \mathbb{R}$ satisfies (1.5), then $C \mu(\tau) \geq$ $\mu(f, \tau)$ for some positive consant $C$. It is easy to check that $\mu(f, \cdot)$ is sub-additive, that is $\mu\left(f, \tau_{1}+\tau_{2}\right) \leq \mu\left(f, \tau_{1}\right)+\mu\left(f, \tau_{2}\right)$ whenever $0 \leq \tau_{1} \leq \tau_{2} \leq \tau_{1}+\tau_{2} \leq 1$. Then, by a result of Efimov ([5], Lemma 4$)$, there exists a concave (hence continuous) nondecreasing function $\mu:[0,1] \rightarrow \mathbb{R}$ such that $\mu(\tau) \leq \mu(f, \tau) \leq 2 \mu(\tau), \tau \in[0,1]$. If 
$f$ is nonconstant, $\mu$ can be chosen to be strictly increasing. Therefore it is natural to make the following

Definition 1. Let $I \subset \mathbb{R}$ be a closed bounded interval, let $\mathcal{B}$ be a Banach space and let $\mu:[0,1] \rightarrow[0,1]$ be a concave strictly increasing function, with $\mu(0)=0$. We say that a function $f: I \rightarrow \mathcal{B}$ is $\mu$-continuos (and we write $f \in \mathcal{C}^{\mu}(I, \mathcal{B})$ ) iff (1.5) is satisfied.

Whenever $f: I \rightarrow \mathcal{B}$ is a continuous function, then certainly $f \in \mathcal{C}^{\mu}(I, \mathcal{B})$ for some concave strictly increasing function $\mu$, with $\mu(0)=0$. Moreover, if $f$ is nonconstant, $\mu$ can be chosen in such a way that $\mu(\tau) \leq \mu(f, \tau) \leq 2 \mu(\tau), \tau \in[0,1]$. If $\mu(\tau)=\tau$, then $\mathcal{C}^{\mu}(I, \mathcal{B})=\operatorname{Lip}(I, \mathcal{B})$; if $\mu(\tau)=\tau^{\alpha}, 0<\alpha<1$, then $\mathcal{C}^{\mu}(I, \mathcal{B})=C^{\alpha}(I, \mathcal{B})$.

Definition 2. Let $\omega:[0,1] \rightarrow \mathbb{R}$ be an nondecreasing function, with $\omega(0)=0$. We say that $\omega$ satisfies the Osgood condition iff

$$
\int_{0}^{1} \frac{1}{\omega(s)} d s=+\infty
$$

Condition (1.6) was introduced by Osgood in [10, while proving uniqueness for ordinary differential equations with non-Lipschitz continuous nonlinearities. If $\omega(\tau)=\tau$, then $\omega$ satisfies the Osgood condition. If $\omega(\tau)=\tau^{\alpha}, 0<\alpha<1$, then $\omega$ does not satisfy the Osgood condition. If $\omega(\tau)=\tau|\log \tau|$, then $\omega$ satisfies the Osgood condition.

The result of Mandache states that if a modulus of continuity $\omega$ does not satisfy the Osgood condition then there exists a parabolic operator of type (1.1) with $m=1$ such that the coefficients are $C^{\infty}$ with respect to $x$ and $C^{\omega}$ with respect to $t$ and the backward uniqueness property does not hold.

Let us come to our result. We shall consider here only operators whose coefficients $\rho_{\alpha \beta}$ 's are independent of the spatial variable $x$. If $m=1$, the general case can be recovered by a microlocal approximation procedure similar to the one exploited in [2], provided the $\rho_{\alpha \beta}$ 's are sufficiently smooth in $x$ (see [4] for details). We do not know whether it is possible to extend the result of [4] to the case $m>1$.

If the coefficients are independent of $x$, the operator $P$ takes the simpler form

$$
P=\partial_{t}+\sum_{0 \leq|\alpha| \leq 2 m} \mathrm{i}^{|\alpha|} \rho_{\alpha}(t) \partial_{x}^{\alpha}
$$

where $\rho_{\alpha} \in \mathbb{R}$ for all $\alpha$. Let $A$ be the set of all $n$-multiindices whose weight is smaller or equal than $2 m$, let $\ell$ be the cardinality of $A$ and let $R:[0, T] \rightarrow \mathbb{R}^{\ell}$, $R(t):=\left(\rho_{\alpha}(t)\right)_{\alpha \in A}$, be a continuous mapping. Setting

$$
\rho_{k}(t, \xi):=(-1)^{k} \frac{\sum_{|\alpha|=k} \rho_{\alpha}(t) \xi^{\alpha}}{|\xi|^{k}}, \quad(t, \xi) \in[0, T] \times\left(\mathbb{R}^{n} \backslash\{0\}\right), \quad k=0, \ldots, 2 m
$$

we assume that the exists $\Lambda>0$ such that, for $(t, \xi) \in[0, T] \times\left(\mathbb{R}^{n} \backslash\{0\}\right)$,

$$
\left|\rho_{k}(t, \xi)\right| \leq \Lambda, \quad k=0, \ldots, 2 m-1, \quad \text { and } \quad 1 / \Lambda \leq \rho_{2 m}(t, \xi) \leq \Lambda .
$$


Consider the following backward-parabolic inequality:

$$
\left\|\partial_{t} u-\sum_{0 \leq|\alpha| \leq 2 m} \mathrm{i}^{|\alpha|} \rho_{\alpha}(t) \partial_{x}^{\alpha} u\right\|_{L^{2}} \leq \tilde{C}\|u\|_{H^{m}} .
$$

The main result of the paper is the following

Theorem 1. Let the modulus of continuity $\mu(R, \cdot)$ of $R(\cdot):=\left(\rho_{\alpha}(\cdot)\right)_{\alpha \in A}$ satisfy the Osgood condition. If $u \in \mathcal{H}_{1}^{m}$ satisfies (1.10) and $u(0, \cdot) \equiv 0$ in $\mathbb{R}^{n}$, then $u \equiv 0$ in $[0, T] \times \mathbb{R}^{n}$.

Notice that, if $R(\cdot)$ is constant, then $R \in \mathcal{C}^{\mu}\left([0, T], \mathbb{R}^{\ell}\right)$ with $\mu(\tau)=\tau$. If $R(\cdot)$ is nonconstant, then we can find a concave strictly increasing function $\mu:[0,1] \rightarrow \mathbb{R}$ such that $\mu(\tau) \leq \mu(R, \tau) \leq 2 \mu(\tau), \tau \in[0,1]$. It follows that in both cases $R \in$ $\mathcal{C}^{\mu}\left([0, T], \mathbb{R}^{\ell}\right)$ for some $\mu$ which satisfies the Osgood condition. This observation is crucial for the proof of Theorem 1 .

Remark 1. Theorem [1 allows to treat also operators with $x$-dependent coefficients up to the order $m$. Indeed, all terms up to the order $m$ are absorbed by the right-hand side of the inequality (1.10).

It is very likely that Theorem 1 be sharp. Indeed not only the example of Mandache confirms it in the case of $m=1$, but, by modifying a well known elliptic counterexample of Pliś [11, we can prove the following

Theorem 2. Let $\mu:[0,1] \rightarrow[0,1]$ be a concave strictly increasing function with $\mu(0)=0$. If $\mu$ does not satisfy the Osgood condition, then for all $m \in \mathbb{N}$ there exist $l \in \mathcal{C}^{\mu}([0,1], \mathbb{R})$, with $1 / 2 \leq l(t) \leq 3 / 2$ for all $t \in[0,1], b_{1}, b_{2}, c \in C_{b}^{\infty}\left([0,1] \times \mathbb{R}^{2}, \mathbb{R}\right)$, and $u \in C_{b}^{\infty}\left([0,1] \times \mathbb{R}^{2}, \mathbb{R}\right)$, with $u(1, \cdot) \equiv 0$ in $\mathbb{R}^{2}$ but $u \neq \equiv 0$ in $[0,1] \times \mathbb{R}^{2}$, such that

$$
\begin{aligned}
& \partial_{t} u+(-1)^{m}\left(\partial_{x_{1}}^{2 m} u+l(t) \partial_{x_{2}}^{2 m} u\right) \\
& \quad+b_{1}(t, x) \partial_{x_{1}} u+b_{2}(t, x) \partial_{x_{2}} u+c(t, x) u=0 \quad \text { in }[0,1] \times \mathbb{R}^{2}
\end{aligned}
$$

Remark 2. If $m=1$, we can take any function $\psi \in C^{\infty}\left(\mathbb{R}^{2}\right)$ such that $\psi(x)=$ $e^{-|x|}$ for $|x| \geq 1$, and defining $v(t, x):=\psi(x) u(t, x)$ we obtain a counterexample to the backward uniqueness property in $\mathcal{H}_{1}^{1}$. However if $m>1$, by the same procedure we get only a non self-adjoint counterexample, with $x$-dependent coefficients up to the order $2 m-1$.

In the next sections we give sketches of the proofs of Theorems 1 and 2.

\section{Proof of Theorem 1$]$}

Theorem 1 is a consequence of the following

Proposition 1. Let $\mu:[0,1] \rightarrow[0,1]$ be a concave strictly increasing function with $\mu(0)=0$. Let $T>0$ and let $R(\cdot) \in \mathcal{C}^{\mu}\left([0, T], \mathbb{R}^{\ell}\right), R(\cdot):=\left(\rho_{\alpha}(\cdot)\right)_{\alpha \in A}$, be a function satisfying (1.8)-(1.9). There exist $C>0, \gamma_{0}>0$ and a strictly increasing $C^{2}$ function $\Phi:\left[0,+\infty\left[\rightarrow\left[0,+\infty\left[\right.\right.\right.\right.$ such that, for all $\gamma \geq \gamma_{0}$ and for all $u \in C_{0}^{\infty}\left(\mathbb{R} \times \mathbb{R}^{n}\right)$ 
with $\operatorname{supp} u \subset[0, T / 2] \times \mathbb{R}^{n}$, the following Carleman estimate holds:

$$
\begin{aligned}
\int_{0}^{T / 2} e^{\frac{2}{\gamma} \Phi(\gamma(T-t))}\left\|\partial_{t} u-\sum_{0 \leq|\alpha| \leq 2 m} \mathrm{i}^{|\alpha|} \rho_{\alpha}(t) \partial_{x}^{\alpha} u\right\|_{L^{2}}^{2} d t & \\
& \geq C \gamma^{1 / 2} \int_{0}^{T / 2} e^{\frac{2}{\gamma} \Phi(\gamma(T-t))}\|u\|_{H^{m}}^{2} d t .
\end{aligned}
$$

Let us briefly sketch how to prove Theorem 1 from the Carleman estimate (2.1). First, we notice that if $\mu(R, \cdot)$ satisfies the Osgood condition then $R \in \mathcal{C}^{\mu}\left([0, T], \mathbb{R}^{\ell}\right)$ for some concave strictly increasing function $\mu$ which satisfies the Osgood condition. Second, by a density argument we have that (2.1) holds for any $u \in \mathcal{H}_{1}^{m}$ such that $u(0, \cdot) \equiv 0$ and $u(t, \cdot) \equiv 0$ for $t \in[T / 2, T]$. Now if $u \in \mathcal{H}_{1}^{m}$ satisfies (1.10) and $u(0, \cdot) \equiv 0$, we take $\vartheta \in C^{\infty}(\mathbb{R}), \vartheta \equiv 0$ on $[T / 2,+\infty], \vartheta \equiv 1$ on $[0, T / 3]$ and we apply (2.1) to the function $\vartheta u$. We obtain

$$
\begin{aligned}
\int_{0}^{T / 2} e^{\frac{2}{\gamma} \Phi(\gamma(T-t))} \| \partial_{t}(\vartheta u)- & \sum_{0 \leq|\alpha| \leq 2 m} \mathrm{i}^{|\alpha|} \rho_{\alpha}(t) \partial_{x}^{\alpha}(\vartheta u) \|_{L^{2}}^{2} d t \\
& \geq C \gamma^{1 / 2} \int_{0}^{T / 2} e^{\frac{2}{\gamma} \Phi(\gamma(T-t))}\|\vartheta u\|_{H^{m}} d t
\end{aligned}
$$

Since $\vartheta \equiv 1$ for $t \in[0, T / 3]$, (1.10) and (2.2) imply

$$
\begin{aligned}
\int_{T / 3}^{T / 2} e^{\frac{2}{\gamma} \Phi(\gamma(T-t))}\left\|\partial_{t}(\vartheta u)-\sum_{0 \leq|\alpha| \leq 2 m} \mathrm{i}^{|\alpha|} \rho_{\alpha}(t) \partial_{x}^{\alpha}(\vartheta u)\right\|_{L^{2}}^{2} d t & \\
& \geq\left(C \gamma^{1 / 2}-\tilde{C}\right) \int_{0}^{T / 3} e^{\frac{2}{\gamma} \Phi(\gamma(T-t))}\|u\|_{H^{m}} d t
\end{aligned}
$$

Since $\Phi$ is increasing, for all sufficiently large $\gamma$ we have

$$
\int_{T / 3}^{T / 2}\left\|\partial_{t}(\vartheta u)-\sum_{0 \leq|\alpha| \leq 2 m} \mathrm{i}^{|\alpha|} \rho_{\alpha}(t) \partial_{x}^{\alpha}(\vartheta u)\right\|_{L^{2}}^{2} d t \geq \frac{C}{2} \gamma^{1 / 2} \int_{0}^{T / 3}\|u\|_{H^{m}}^{2} d t .
$$

Letting $\gamma \rightarrow \infty$, we get $u \equiv 0$ in $[0, T / 3] \times \mathbb{R}^{n}$. Finally, a standard connection argument implies that $u \equiv 0$ in $[0, T] \times \mathbb{R}^{n}$.

Let us come to the proof of Lemma 1] Let $\Phi:[0,+\infty[\rightarrow[0,+\infty[$ be of class $C^{2}$ and increasing. Setting $v(t, x):=e^{\frac{1}{\gamma} \Phi(\gamma(T-t))} u(t, x)$ and denoting by $\hat{v}(t, \xi)$ the Fourier transform of $v(t, x)$ with respect to $x$, (2.1) becomes

$$
\begin{array}{r}
\int_{0}^{T / 2} \int_{\mathbb{R}^{n}}\left|\partial_{t} \hat{v}(t, \xi)-\left(\sum_{k=0}^{2 m} \rho_{k}(t, \xi)|\xi|^{k}-\Phi^{\prime}(\gamma(T-t))\right) \hat{v}(t, \xi)\right|^{2} d \xi d t \\
\geq C \gamma^{1 / 2} \int_{0}^{T / 2} \int_{\mathbb{R}^{n}}\left(|\xi|^{2 m}|\hat{v}(t, \xi)|^{2}+|\hat{v}(t, \xi)|^{2}\right) d \xi d t
\end{array}
$$


Denoting by $\Xi$ the left member of (2.5), direct computation and integration by parts give

$$
\begin{aligned}
\Xi & =\int_{0}^{T / 2} \int_{\mathbb{R}^{n}}\left|\partial_{t} \hat{v}(t, \xi)\right|^{2} d \xi d t \\
& +\left.\int_{0}^{T / 2} \int_{\mathbb{R}^{n}}\left|\sum_{k=0}^{2 m} \rho_{k}(t, \xi)\right| \xi\right|^{k}-\left.\Phi^{\prime}(\gamma(T-t))\right|^{2}|\hat{v}(t, \xi)|^{2} d \xi d t \\
& +\int_{0}^{T / 2} \int_{\mathbb{R}^{n}} \gamma \Phi^{\prime \prime}(\gamma(T-t))|\hat{v}(t, \xi)|^{2} d \xi d t \\
& -2 \operatorname{Re} \int_{0}^{T / 2} \int_{\mathbb{R}^{n}} \partial_{t} \hat{v}(t, \xi)\left(\sum_{k=0}^{2 m} \rho_{k}(t, \xi)|\xi|^{k}\right) \overline{\hat{v}(t, \xi)} d \xi d t .
\end{aligned}
$$

If the $\rho_{k}(\cdot, \xi)$ 's are Lipschitz continuos (that is: if $R(\cdot)$ is Lipschitz continuos), one could just take $\Phi(\tau):=\tau^{2}$, integrate by parts the double product and get the desired estimate (see [7] for details). If the $\rho_{k}(\cdot, \xi)$ 's are not Lipschitz continuos, we exploit a standard approximation procedure. We extend $\rho_{k}(\cdot, \xi)$ on the whole $\mathbb{R}$, we take $\phi \in C_{0}^{\infty}(\mathbb{R})$ such that $\int_{\mathbb{R}} \phi(s) d s=1, \phi \geq 0$ and $\operatorname{supp} \phi \subset[-1 / 2,1 / 2]$, and then we define

$$
\rho_{k, \epsilon}(t, \xi):=\int_{\mathbb{R}} \rho_{k}(s, \xi) \frac{1}{\epsilon} \phi\left(\frac{t-s}{\epsilon}\right) d s, \quad(t, \xi) \in \mathbb{R} \times\left(\mathbb{R}^{n} \backslash\{0\}\right) .
$$

It follows that $\rho_{k, \epsilon}(\cdot, \xi) \in C^{\infty}$ for every $\xi \in \mathbb{R}^{n} \backslash\{0\}$. Moreover

$$
\left|\rho_{k, \epsilon}(t, \xi)-\rho_{k}(t, \xi)\right| \leq K \mu(\epsilon), \quad(t, \xi) \in \mathbb{R} \times\left(\mathbb{R}^{n} \backslash\{0\}\right)
$$

and

$$
\left|\rho_{k, \epsilon}^{\prime}(t, \xi)\right| \leq K \frac{\mu(\epsilon)}{\epsilon}, \quad(t, \xi) \in \mathbb{R} \times\left(\mathbb{R}^{n} \backslash\{0\}\right)
$$

(here "I" indicates derivation with respect to $t$ ). Now let $\epsilon_{1}, \ldots, \epsilon_{2 m}$ be approximation parameters to be chosen later. Then, adding and subtracting $\rho_{k, \epsilon_{k}}$ and integrating by parts with respect to $t$, we get:

$$
\begin{aligned}
& -2 \operatorname{Re} \int_{0}^{T / 2} \int_{\mathbb{R}^{n}} \partial_{t} \hat{v}(t, \xi)\left(\sum_{k=0}^{2 m} \rho_{k}(t, \xi)|\xi|^{k}\right)|\xi|^{2} \overline{\hat{v}(t, \xi)} d \xi d t \\
& \geq-\int_{0}^{T / 2} \int_{\mathbb{R}^{n}}\left|\partial_{t} \hat{v}(t, \xi)\right|^{2} d \xi d t-K \int_{0}^{T / 2} \int_{\mathbb{R}^{n}}\left(\sum_{k=0}^{2 m} \frac{\mu\left(\epsilon_{k}\right)}{\epsilon_{k}}|\xi|^{k}\right)|\hat{v}(t, \xi)|^{2} d \xi d t \\
& -K^{2} \int_{0}^{T / 2} \int_{\mathbb{R}^{n}}\left(\sum_{k=0}^{2 m} \mu\left(\epsilon_{k}\right)^{2}|\xi|^{2 k}\right)|\hat{v}(t, \xi)|^{2} d \xi d t
\end{aligned}
$$

Now the first key idea is to let the approximation parameters $\epsilon_{k}$ depend on $\xi$ (cf [1]). First, we observe that, by (1.9), there exist $N_{0} \geq 1$ and $\Lambda_{0}>0$ such that, for 
all $|\xi| \geq N_{0}$

$$
\frac{1}{\Lambda_{0}}|\xi|^{2 m} \leq \sum_{k=0}^{2 m} \rho_{k}(t, \xi)|\xi|^{k} \leq \Lambda_{0}|\xi|^{2 m}
$$

Then we take

$$
\epsilon_{k}:= \begin{cases}|\xi|^{-k} & \text { if }|\xi| \geq N_{0} \\ N_{0}^{-k} & \text { if }|\xi| \leq N_{0}\end{cases}
$$

With this choice, noticing also that $s^{2} \mu(1 / s)$ is increasing on $[1,+\infty]$, we obtain that there exists a positive constant $\tilde{K}$ such that:

$$
\begin{aligned}
& \int_{0}^{T / 2} \int_{\mathbb{R}^{n}}\left|\partial_{t} \hat{v}(t, \xi)-\left(\sum_{k=0}^{2 m} \rho_{k}(t, \xi)|\xi|^{k}-\Phi^{\prime}(\gamma(T-t))\right) \hat{v}(t, \xi)\right|^{2} d \xi d t \\
& \geq \gamma \int_{0}^{T / 2} \int_{\mathbb{R}^{n}} \Phi^{\prime \prime}(\gamma(T-t))|\hat{v}(t, \xi)|^{2} d \xi d t \\
& +\left.\int_{0}^{T / 2} \int_{\mathbb{R}^{n}}\left|\sum_{k=0}^{2 m} \rho_{k}(t, \xi)\right| \xi\right|^{k}-\left.\Phi^{\prime}(\gamma(T-t))\right|^{2}|\hat{v}(t, \xi)|^{2} d \xi d t \\
& -\tilde{K} \int_{0}^{T / 2} \int_{\mathbb{R}^{n}}|\hat{v}(t, \xi)|^{2} d \xi d t \\
& -\tilde{K} \int_{0}^{T / 2} \int_{\left\{|\xi| \geq N_{0}\right\}}^{\mu\left(1 /|\xi|^{2 m}\right)|\xi|^{4 m}|\hat{v}(t, \xi)|^{2} d \xi d t}
\end{aligned}
$$

Now we observe that:

- the summand $(i)$ behaves well, provided $\Phi^{\prime \prime}(\tau) \geq 1$ for large $\tau$;

- the same condition let the summand (iii) be absorbed by $(i)$;

- when $\left.|\xi|^{2 m} \geq 2 \Lambda_{0} \Phi^{\prime}(\gamma(T-t))\right)$, then the integrand in (ii) behaves like $|\xi|^{4 m}$, which is enough to compensate the integrand in $(i v)$;

- when $\left.N_{0}^{2 m} \leq|\xi|^{2 m} \leq\left(1 / 2 \Lambda_{0}\right) \Phi^{\prime}(\gamma(T-t))\right)$, then again the integrand in $(i i)$ behaves like $|\xi|^{4 m}$, which is enough to compensate the integrand in $(\mathrm{iv})$;

- the difficult case is when $|\xi|^{2 m} \sim \Phi^{\prime}(\gamma(T-t))$.

At this point the second key idea is to modulate the weight $\Phi$ on the function $\mu$ (cf Tarama [12]). Roughly speaking, we ask that, when $|\xi|^{2 m} \sim \Phi^{\prime}(\gamma(T-t)$ ), then the integrand in $(i v)$ must be compensated by the integrand in $(i)$. More precisely, we ask that $\Phi^{\prime \prime}(\gamma(T-t)) \sim \mu\left(1 /|\xi|^{2 m}\right)|\xi|^{4 m}$. In other words, the Carleman estimates (2.1) will follow, provided $\Phi$ satisfies the ordinary differential equation

$$
\Phi^{\prime \prime}=\mu\left(1 / \Phi^{\prime}\right)\left(\Phi^{\prime}\right)^{2} \text {. }
$$

All we have to do then is to find a solution of (2.12) and to check that:

- $\Phi$ is defined on $[0+\infty[$, i.e. it does not blow up in finite time;

- $\Phi$ is positive and increasing;

- $\Phi^{\prime \prime}(\tau) \geq 1$ for all sufficiently large $\tau$. 
Equation (2.12) can be easily solved by separation of variables. The explicit solution of the Cauchy problem with initial values $\Phi(0)=0$ and $\Phi^{\prime}(0)=1$ is given by:

$$
\begin{array}{rlrl}
\eta(t) & :=\int_{1 / t}^{1} \frac{1}{\mu(s)} d s, & & t \geq 1 \\
\Phi(\tau):=\int_{0}^{\tau} \eta^{-1}(r) d r, & \tau \geq 0
\end{array}
$$

The Osgood condition precisely guarantees that $\Phi$ is defined on $[0,+\infty[$. The other properties that we require for $\Phi$ follow by easy computation. With this choice of $\Phi$ we finally get the desired Carleman estimate (2.1). The details are left to the reader.

\section{Proof of Theorem 2}

The proof of Theorem 2 is very similar to that one of Theorem 3 in [4. Also in this case we will follow closely the construction of the example in [11]. Let $A, B, C$, $J$ be four $C^{\infty}$ functions defined in $\mathbb{R}$ with $0 \leq A(s), B(s), C(s) \leq 1,-2 \leq J(s) \leq 2$ for all $s \in \mathbb{R}$ and

$$
\begin{array}{lll}
A(s)=1 \quad \text { for } s \leq \frac{1}{5}, & A(s)=0 & \text { for } s \geq \frac{1}{4}, \\
B(s)=0 & \text { for } s \leq 0 \text { or } s \geq 1, & B(s)=1 \quad \text { for } \frac{1}{6} \leq s \leq \frac{1}{2}, \\
C(s)=0 \quad \text { for } s \leq \frac{1}{4}, & C(s)=1 \quad \text { for } s \geq \frac{1}{3}, \\
J(s)=-2 \quad \text { for } s \leq \frac{1}{6} \text { or } s \geq \frac{1}{2}, & J(s)=2 \text { for } \frac{1}{5} \leq s \leq \frac{1}{3} .
\end{array}
$$

Let $\left(a_{n}\right)_{n},\left(z_{n}\right)_{n}$ be two real sequences such that

$$
\begin{aligned}
-1<a_{n}<a_{n+1} & \text { for all } n \geq 1, \quad \lim _{n} a_{n}=0, \\
1<z_{n}<z_{n+1} & \text { for all } n \geq 1, \quad \lim _{n} z_{n}=+\infty ;
\end{aligned}
$$

and let us define $r_{n}=a_{n+1}-a_{n}, q_{1}=0, q_{n}=\sum_{k=2}^{n} z_{k} r_{k-1}$ for all $n \geq 2$, and $p_{n}=\left(z_{n+1}-z_{n}\right) r_{n}$. We suppose moreover that

$$
p_{n}>1 \text { for all } n \geq 1 \text {. }
$$

We set $A_{n}(t)=A\left(\frac{t-1-a_{n}}{r_{n}}\right), B_{n}(t)=B\left(\frac{t-1-a_{n}}{r_{n}}\right), C_{n}(t)=C\left(\frac{t-1-a_{n}}{r_{n}}\right)$ and $J_{n}(t)=$ $J\left(\frac{t-1-a_{n}}{r_{n}}\right)$. We define

$$
\begin{aligned}
& v_{n}\left(t, x_{1}\right)=\exp \left(-q_{n}-z_{n}\left(t-1-a_{n}\right)\right) \cos \sqrt[2 m]{z_{n}} x_{1} \\
& w_{n}\left(t, x_{2}\right)=\exp \left(-q_{n}-z_{n}\left(t-1-a_{n}\right)+J_{n}(t) p_{n}\right) \cos \sqrt[2 m]{z_{n}} x_{2}
\end{aligned}
$$


and

$$
\begin{aligned}
& u\left(t, x_{1}, x_{2}\right) \\
& = \begin{cases}v_{1}\left(t, x_{1}\right) & \text { for } 0 \leq t \leq 1+a_{1}, \\
A_{n}(t) v_{n}\left(t, x_{1}\right)+B_{n}(t) w_{n}\left(t, x_{2}\right) & \\
+C_{n}(t) v_{n+1}\left(t, x_{1}\right) & \text { for } 1+a_{n} \leq t \leq 1+a_{n+1}, \\
0 & \text { for } t=1 .\end{cases}
\end{aligned}
$$

If for all $\alpha, \beta \gamma>0$

$$
\lim _{n} \exp \left(-q_{n}+2 p_{n}\right) z_{n+1}^{\alpha} p_{n}^{\beta} r_{n}^{-\gamma}=0
$$

then $u$ is a $C_{b}^{\infty}\left([0,1] \times \mathbb{R}^{2}, \mathbb{R}\right)$ function. We define

$$
l(t)= \begin{cases}1 & \text { for } t \leq 1+a_{1} \text { or } t=1 \\ 1+J_{n}^{\prime}(t) p_{n} z_{n}^{-1} & \text { for } 1+a_{n} \leq t \leq 1+a_{n+1} .\end{cases}
$$

The condition

$$
\sup _{n}\left\{p_{n} r_{n}^{-1} z_{n}^{-1}\right\} \leq \frac{1}{2\left\|J^{\prime}\right\|_{L^{\infty}}}
$$

guarantees that the operator $E=\partial_{t}+(-1)^{m}\left(\partial_{x_{1}}^{2 m}-l(t) \partial_{x_{2}}^{2 m}\right)$ is parabolic. Moreover $l$ is a $C^{\mu}$ function under the condition

$$
\sup _{n}\left\{\frac{p_{n} r_{n}^{-1} z_{n}^{-1}}{\mu\left(r_{n}\right)}\right\}<+\infty
$$

Finally we define

$$
\begin{aligned}
& b_{1}=-\frac{E u}{u^{2}+\left(\partial_{x_{1}} u\right)^{2}+\left(\partial_{x_{2}} u\right)^{2}} \partial_{x_{1}} u, \\
& b_{2}=-\frac{E u}{u^{2}+\left(\partial_{x_{1}} u\right)^{2}+\left(\partial_{x_{2}} u\right)^{2}} \partial_{x_{2}} u, \\
& c=-\frac{E u}{u^{2}+\left(\partial_{x_{1}} u\right)^{2}+\left(\partial_{x_{2}} u\right)^{2}} u .
\end{aligned}
$$

As in 11], or similarly in [4, the coefficients $b_{1}, b_{2}, c$ will be in $C_{b}^{\infty}$ if for all $\alpha, \beta$, $\gamma>0$

$$
\lim _{n} \exp \left(-p_{n}\right) z_{n+1}^{\alpha} p_{n}^{\beta} r_{n}^{-\gamma}=0
$$

We choose

$$
a_{n}=-\sum_{j=n}^{+\infty} \frac{1}{\left(j+k_{0}\right)^{2} \mu\left(\frac{1}{j+k_{0}}\right)}, \quad z_{n}=\left(n+k_{0}\right)^{3}
$$

with $k_{0}$ sufficiently large.

To conclude the proof it will be sufficient to verify in the same way than in 4] that, with the choice (3.8), the conditions (3.1),..., (3.7) hold. We let it to the reader. 


\section{REFERENCES}

[1] F. Colombini, E. De Giorgi, S. Spagnolo, Sur les équations hyperboliques avec des coefficients qui ne dépendent que du temps, Ann. Scuola Norm. Sup. Pisa Cl. Sci. 6 (1979), 511-559.

[2] F. Colombini, N. Lerner, Hyperbolic operators having non-Lipschitz coefficients, Duke Math. J. 77 (1995), 657-698.

[3] C. Bardos, L. Tartar, Sur l'unicité rétrograde des équations paraboliques et quelques questions voisines, Arch. Rat. Mech. Anal. 50 (1973), 10-25.

[4] D. Del Santo and M. Prizzi, Backward uniqueness for parabolic operators whose coefficients are non-Lipschitz continuous in time, J. Math. Pures Appl. 84 (2005), 471-491.

[5] A.V. Efimov, Linear methods of approximation of continuous periodic functions, Math. Sbornik 54 (1961), 51-90 (in Russian).

[6] J.-M. Ghidaglia, Some backward uniqueness results, Nonlinear Analysis TMA 10 (1986), $777-790$.

[7] J.-L. Lions, B. Malgrange, Sur l'unicité rétrograde dans les problèmes mixtes paraboliques, Math. Scand. 8 (1960), 277-286.

[8] N. Mandache, On a counterexample concerning unique continuation for elliptic equations in divergence form, Math. Phys. Anal. Geom. 1 (1998), 273-292.

[9] K. Miller, Nonunique continuation for uniformly parabolic and elliptic equations in self-adjoint divergence form with Hölder continuous coefficients, Arch. Rational Mech. Anal. 54 (1974), $105-117$.

[10] W.F. Osgood, Beweis der Existenz einer Lösung der Differentialgleichung $d y / d x=f(x, y)$ ohne Hinzunahme der Cauchy-Lipschitz'schen Bedingung, Monatsh. Math. Phys. 9 (1898), 331-345.

[11] A. Pliś, On non-uniqueness in Cauchy problem for an elliptic second order differential equation, Bull. Ac. Pol. Sci. 11 (1963), 95-100.

[12] S. Tarama, Local uniqueness in the Cauchy problem for second order elliptic equations with non-Lipschitzian coefficients, Publ. Res. Inst. Math. Sci. 33 (1997), no. 1, 167-188.

[13] A. Tychonoff, Théorème d'unicité pour l'équation de la chaleur, Math. Sbornik 42 (1935), $199-215$

Daniele Del Santo, Università di Trieste, Dipartimento di Matematica e Informatica, Via Valerio 12/1, 34127 Trieste, Italy

E-mail address: delsanto@univ.trieste.it

Martino Prizzi, Università di Trieste, Dipartimento di Matematica e Informatica, Via Valerio 12/1, 34127 Trieste, Italy

E-mail address: prizzi@dsm.univ.trieste.it 